\title{
Block-diagonal characterization of locally finite simple groups of $p$-type
}

\author{
Stefaan Delcroix \\ Communicated by Robert M. Guralnick
}

\begin{abstract}
In this paper, we prove the following characterization of LFS-groups of $p$-type:
Let $G$ be a locally finite group and $\delta$ be a local system for $G$ such that $\delta \subseteq \mathcal{P}$ and for all $S \in \mathcal{8}:$

(i) $\left\{T \in \mathcal{S}(S) \mid V_{T}^{j}\right.$ is block-diagonal for $S$ for $\left.j=1, \ldots, n_{T}\right\}$ is a local system for $G$.

(ii) there exists $F_{S} \in \mathcal{S}(S)$ such that
\end{abstract}

(a) $V_{F_{S}}^{j}$ is block-diagonal for $S$ for $j=1, \ldots, n_{F_{S}}$,

(b) $\left[V_{F_{S}}^{j}, S_{i}^{*}\right] \neq 0$ for $i=1, \ldots, n_{S}$ and $j=1, \ldots, n_{F_{S}}$,

(c) for $j=1, \ldots, n_{F_{S}}$,

$$
\sum_{i=1}^{n_{S}} n_{S F_{S}}^{i j} \operatorname{dim}_{K_{F_{S}}^{j}}\left\langle K_{S}^{i}, K_{F_{S}}^{j}\right\rangle \geq 2
$$

where $n_{S F_{S}}^{i j}$ is the number of composition factors $C$ for $\hat{S}$ on $V_{F_{S}}^{j}$ with $\left[C, S_{i}^{*}\right] \neq 0$.

Then $G / \operatorname{LSol}(G)$ is an LFS-group of p-type and $[G, \operatorname{LSol}(G)] \leq O_{p}(G)$.

We use this theorem to construct a general family of LFS-groups of $p$-type.

\section{Introduction, definitions and notations}

The locally finite simple groups have been divided into four types: finitary, 1-type, $p$-type and $\infty$-type (see $[3,5])$. The groups of finitary type are those in which elements exhibit 'no growth' (for an appropriate definition) and have been classified. In the groups of $\infty$-type, all the elements exhibit 'unrestricted growth'. The groups of 1-type and $p$-type sit in between: the elements exhibit 'linear growth'. These groups (1-type and $p$-type) are the groups of diagonal type because subgroups are embedded in each other in a specific way ('diagonally'). In the groups of 1-type, these subgroups involve alternating groups while in the groups of $p$-type, they involve classical groups in characteristic $p$.

In [1, Section 4.15], the author proved that every group of $p$-type admits a Kegel cover whose members modulo normal $p$-subgroups are direct products of classical groups in characteristic $p$ and the embeddings are diagonal. In this paper, 
we prove the converse: a locally finite group with a subgroup cover as described above must be simple of $p$-type modulo a locally solvable normal subgroup. We will use this converse to construct a general family of groups of $p$-type.

We finish this introduction with some definitions and notations.

Let $G$ be a group. Then $G$ is locally finite if every finite subset of $G$ generates a finite subgroup of $G$. The set $\mathscr{L}=\left\{H_{i} \mid i \in I\right\}$ is a local system for $G$ if $H_{i}$ is a finite subgroup of $G$ for all $i \in I$ such that $\bigcup_{i \in I} H_{i}=G$ and for all $i, j \in I$, there exists $k \in I$ with $H_{i}, H_{j} \leq H_{k}$. We say that $G$ is an LFS-group if $G$ is an infinite, locally finite, simple group. The set $\mathcal{K}=\left\{\left(H_{i}, M_{i}\right) \mid i \in I\right\}$ is a Kegel cover for $G$ if $H_{i}$ is a finite subgroup of $G$ and $M_{i}$ is a maximal normal subgroup of $H_{i}$ for all $i \in I$ such that for every finite subgroup $F$ of $G$, there exists $i \in I$ with $F \leq H_{i}$ and $F \cap M_{i}=1$. The groups $\left\{H_{i} / M_{i} \mid i \in I\right\}$ are called the factors of the Kegel cover. Note that by [4, p. 113], every LFS-group has a Kegel cover.

Let $G$ be an LFS-group. Then $G$ is finitary if there exists a field $K$ and a faithful $K G$-module $V$ such that $[V, g]:=\left\{v^{g}-v \mid v \in V\right\}$ is finite-dimensional for all $g \in G$. We say that $G$ is of $p$-type for some prime $p$ if $G$ is not finitary and every Kegel cover for $G$ contains at least one factor that is isomorphic to a classical group defined over a field of characteristic $p$.

If $g$ acts on a $K$-vector space $V$, then we put

$$
\operatorname{deg}_{V}(g)=\operatorname{dim}_{K}[V, g] \text { and } \operatorname{pdeg}_{V}(g)=\min \left\{\operatorname{deg}_{V}(\lambda g) \mid \lambda \in K\right\} .
$$

If $G$ is a finite group, we denote by $G^{\infty}$ the largest perfect subgroup of $G$. We refer to $G^{\infty}$ as the perfect part of $G$.

If $S$ is a finite perfect group, we denote by $\hat{S}$ the universal central extension of $S$ and by $\varphi_{S}: \hat{S} \rightarrow S$ a fixed epimorphism (note that $\operatorname{Ker} \varphi_{S} \leq Z(\hat{S})$ ).

If $G$ is a group, $S \leq G$ and $\mathscr{L}$ is a collection of subgroups of $G$, then we put $\mathscr{L}(S)=\{L \in \mathscr{L} \mid S \leq L\}$.

For the rest of this paper, let $p$ be a fixed prime. We denote by $\mathcal{P}$ the class of all finite perfect groups $S$ such that $S / O_{p}(S)=S^{1} \ldots S^{n_{S}}$ where

(1) $S^{i}$ is a component of $S / O_{p}(S)$ for $i=1, \ldots, n_{S}$,

(2) $S^{i} / Z\left(S^{i}\right)$ is isomorphic to a classical group defined over a field of characteristic $p$ for $i=1, \ldots, n_{S}$.

Fix $1 \leq i \leq n_{S}$. We put $P G_{S}^{i}=S^{i} / Z\left(S^{i}\right)$, which is a classical group over the field $K_{S}^{i}$ and the $K_{S}^{i}$-vector space $V_{S}^{i}$, so

$$
P G_{S}^{i} \in\left\{\mathrm{PSL}_{K_{S}^{i}}\left(V_{S}^{i}\right), \mathrm{PSp}_{K_{S}^{i}}\left(V_{S}^{i}\right), \mathrm{PSU}_{K_{S}^{i}}\left(V_{S}^{i}\right), \mathrm{P} \Omega_{K_{S}^{i}}\left(V_{S}^{i}\right)\right\} .
$$

We denote the linear classical group by $G_{S}^{i}$, so

$$
G_{S}^{i} \in\left\{\mathrm{SL}_{S_{S}^{i}}\left(V_{S}^{i}\right), \operatorname{Sp}_{K_{S}^{i}}\left(V_{S}^{i}\right), \mathrm{SU}_{K_{S}^{i}}\left(V_{S}^{i}\right), \Omega_{K_{S}^{i}}\left(V_{S}^{i}\right)\right\} .
$$


Let $S_{i}$ be the perfect part of the preimage in $S$ of $S^{i}$ and let $S_{i}^{*}$ be the perfect part of $S_{i}^{\varphi}{ }_{S}^{-1}$. Put

$$
\pi_{S}^{i}: G_{S}^{i} \rightarrow P G_{S}^{i}: x \rightarrow x Z\left(G_{S}^{i}\right)
$$

and

$$
\alpha_{S}^{i}: S \rightarrow P G_{S}^{i}: s_{1} \ldots s_{n_{S}} \rightarrow s_{i} \operatorname{Sol}\left(S_{i}\right)
$$

where $s_{j} \in S_{j}$ for $j=1, \ldots, n_{S}$. Then there exists a unique epimorphism

$$
\beta_{S}^{i}: \hat{S} \rightarrow G_{S}^{i}
$$

such that $\beta_{S}^{i} \pi_{S}^{i}=\varphi_{S} \alpha_{S}^{i}$.

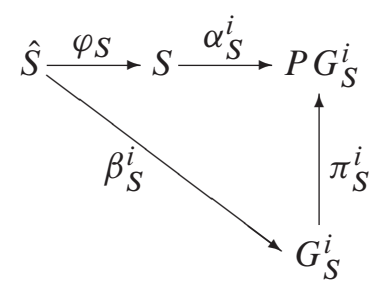

Let $K$ be a field and $V$ be a finite-dimensional $K$-vector space. We denote the dual of $V$ by $V^{*}$. For $\alpha \in \operatorname{Aut}(K)$, we define a new scalar multiplication ${ }^{\alpha} \alpha$ on $V$ by putting $v \cdot{ }_{\alpha} k=v k^{\alpha}$ for all $v \in V$ and all $k \in K$; we will write $V^{\alpha}$ to show that we are working with the scalar multiplication $\cdot \alpha$.

We fix an algebraic closure $\overline{\operatorname{GF}(p)}$ of $\operatorname{GF}(p)$ (in general, $\operatorname{GF}(q)$ is the Galoisfield with $q$ elements). For any finite field $K$ with char $K=p$, we fix an embedding from $K$ into $\overline{\mathrm{GF}(p)}$ (so we view $K$ as a subfield of $\overline{\mathrm{GF}(p)}$ ). If $K$ and $L$ are finite fields, we will denote by $\langle K, L\rangle$ the subfield of $\overline{\mathrm{GF}(p)}$ generated by $K$ and $L$.

Definition 1.1. Let $S \in \mathcal{P}$, let $K$ be a finite field with char $K=p$, let $V$ be a finite-dimensional $K$-vector space and let $\theta: S \rightarrow \operatorname{PSL}_{K}(V)$ be a homomorphism. Put $\pi: \operatorname{SL}_{K}(V) \rightarrow \operatorname{PSL}_{K}(V), x \rightarrow x Z\left(\operatorname{SL}_{K}(V)\right)$. Then there exists a unique epimorphism $\hat{\theta}: \hat{S} \rightarrow \operatorname{SL}_{K}(V)$ such that $\hat{\theta} \pi=\varphi_{S} \theta$. We say that $V$ is block-diagonal for $S$ if for every non-trivial $K$-composition factor $C$ for $S$ on $V$ (see the diagrams below)

(i) there exists exactly one $i \in\left\{1, \ldots, n_{S}\right\}$ such that $S_{i}$ is not trivial on $C$ (or equivalent, $\left.\left[C, S_{i}^{*}\right] \neq 0\right)$.

(ii) for this $i$, there exists $\sigma \in \operatorname{Aut}\left(K_{S}^{i}\right)$ such that

$$
C \cong\left(V_{S}^{i}\right)^{\sigma} \otimes_{K_{S}^{i}} L \quad \text { or } \quad C \cong\left(V_{S}^{i}\right)^{*, \sigma} \otimes_{K_{S}^{i}} L
$$

as $K \hat{S}$-modules where $L=\left\langle K, K_{S}^{i}\right\rangle$. 

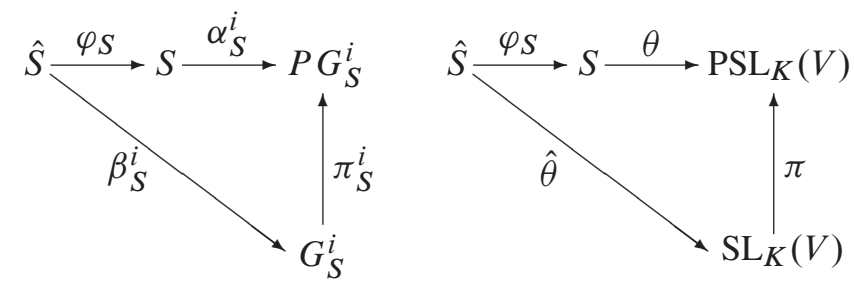

In [1, Section 4.15], the author proved that every LFS-group of $p$-type has a Kegel cover $\mathcal{K}$ such that $S \in \mathcal{P}$ for all $(S, M) \in \mathcal{K}$ and $V_{T}^{i}$ is block-diagonal for $S$ for all $(S, M),(T, N) \in \mathcal{K}$ with $S \leq T$ and all $1 \leq i \leq n_{T}$. In this paper, we prove more or less the converse.

\section{Block-diagonal characterization of LFS-groups of $p$-type}

In [5, Section 3.3], U. Meierfrankenfeld proved that if $G$ is an LFS-group of $p$-type, then $G$ has a Kegel cover $\mathcal{K}$ such that $H \in \mathcal{P}$ for all $(H, M) \in \mathcal{K}$. The converse however is not true. There exist non-finitary LFS-groups with such a Kegel cover that are not of $p$-type; P. Hall's universal group is an example. Blockdiagonality however is something typical for groups of $p$-type.

In this section, we prove the following theorem:

Theorem 2.1. Let $G$ be a locally finite group and $\&$ be a local system for $G$ such that $\delta \subseteq \mathcal{P}$ and for all $S \in \mathcal{8}$ :

(i) $\left\{T \in \mathcal{S}(S) \mid V_{T}^{j}\right.$ is block-diagonal for $S$ for $\left.j=1, \ldots, n_{T}\right\}$ is a local system for $G$.

(ii) there exists $F_{S} \in \delta(S)$ such that

(a) $V_{F_{S}}^{j}$ is block-diagonal for $S$ for $j=1, \ldots, n_{F_{S}}$,

(b) $\left[V_{F_{S}}^{j}, S_{i}^{*}\right] \neq 0$ for $i=1, \ldots, n_{S}$ and $j=1, \ldots, n_{F_{S}}$.

(c) for $j=1, \ldots, n_{F_{S}}$,

$$
\sum_{i=1}^{n_{S}} n_{S F_{S}}^{i j} \operatorname{dim}_{K_{F_{S}}^{j}}\left\langle K_{S}^{i}, K_{F_{S}}^{j}\right\rangle \geq 2
$$

where for $1 \leq i \leq n_{S}$ and $1 \leq j \leq n_{F_{S}}$, we denote by $n_{S F_{S}}^{i j}$ the number of composition factors $C$ for $\hat{S}$ on $V_{F_{S}}^{j}$ with $\left[C, S_{i}^{*}\right] \not 0$; note that $n_{S F_{S}}^{i j} \geq 1$ by (b) since $S_{i}^{*}$ is perfect.

Then $G / \operatorname{LSol}(G)$ is an LFS-group of $p$-type and $[G, \operatorname{LSol}(G)] \leq O_{p}(G)$.

Note that conditions (i) and (ii) (a)-(b) imply that $G$ is a direct limit of subgroups in which classical groups act non-trivially and block-diagonally. Further, 
condition (ii) (c) is critical as it forces $G / \operatorname{LSol}(G)$ to be of $p$-type. Without this condition, $G / \operatorname{LSol}(G)$ would be finitary.

Before proving this theorem, we provide an example on how this theorem can be used to construct LFS-groups of $p$-type. The next section will include a general construction of LFS-groups of $p$-type.

Let $K$ be a finite field with char $K=p$ and $V_{0}$ be a finite-dimensional $K$-vector space such that $\mathrm{SL}_{K}\left(V_{0}\right)$ is perfect. Put $n=\operatorname{dim}_{K} V_{0}$. For $i \geq 1$, define

$$
V_{i}=V_{i-1} \oplus V_{i-1} \text {. }
$$

Fix a $K$-basis $\beta$ for $V_{0}$. Then $\beta$ induces in a natural way a $K$-basis for $V_{i}$ for $i \geq 1$. All matrix representations are with respect to these bases. For $i \geq 1$, put

$$
G_{i}=\left\{\left[\begin{array}{cc}
A & 0 \\
X & B
\end{array}\right] \mid A, B \in \mathrm{SL}_{K}\left(V_{i-1}\right), X \in K^{2^{i-1} n \times 2^{i-1} n}\right\} \leq \mathrm{SL}_{K}\left(V_{i}\right) .
$$

For $g \in G_{i}$, put

$$
g=\left[\begin{array}{cc}
A_{g, 1} & 0 \\
X_{g} & A_{g, 2}
\end{array}\right]
$$

For $i \geq 1$, consider the embedding

$$
\varphi_{i}: G_{i} \rightarrow G_{i+1}:\left[\begin{array}{cc}
A & 0 \\
X & B
\end{array}\right] \rightarrow\left[\begin{array}{cccc}
A & 0 & 0 & 0 \\
X & B & 0 & 0 \\
0 & 0 & A & 0 \\
X & 0 & X & B
\end{array}\right] .
$$

Let $G$ be the direct limit of the $G_{i}$. We view $G_{i}$ as a subgroup of $G$ for $i \geq 1$ (and so $G_{i} \leq G_{i+1}$ ). Then $G$ is an infinite locally finite group and $\left\{G_{i} \mid i \geq 1\right\}$ is a local system for $G$.

Fix $i \geq 1$. Put $S=G_{i}$.

First, we show that $S \in \mathcal{P}$. Put $m=2^{i-1} n \times 2^{i-1} n$. Put

$$
S_{1}=\left\{s \in S \mid A_{s, 2}=I\right\} \quad \text { and } \quad S_{2}=\left\{s \in S \mid A_{s, 1}=I\right\} .
$$

Pick $A \in \mathrm{SL}_{K}\left(V_{i-1}\right)$ and $X \in K^{m \times m}$. Pick a matrix $B \in \mathrm{SL}_{K}\left(V_{i-1}\right)$ such that $\operatorname{det}(B-I) \neq 0$ (we can do this by choosing $B$ such that its characteristic polynomial is $\left.(-1)^{m}\left(X^{m}-X^{m-1}+(-1)^{m}\right)\right)$. Since $\operatorname{SL}_{K}\left(V_{i-1}\right) \cong\left\{s \in S_{1} \mid X_{s}=0\right\}$ and $\mathrm{SL}_{K}\left(V_{i-1}\right)$ is perfect, there exists $s \in S_{1}^{\prime}$ with $A_{s, 1}=A$ and $X_{s}=0$. Then

$$
\left[\begin{array}{cc}
A & 0 \\
0 & I
\end{array}\right]\left[\left[\begin{array}{cc}
B^{-1} & 0 \\
X(B-I)^{-1} & I
\end{array}\right],\left[\begin{array}{cc}
B & 0 \\
0 & I
\end{array}\right]\right]=\left[\begin{array}{cc}
A & 0 \\
X & I
\end{array}\right] \in S_{1}^{\prime} .
$$


So $S_{1}^{\prime}=S_{1}$. Similarly, $S_{2}^{\prime}=S_{2}$. Since

$$
\left[\begin{array}{ll}
A & 0 \\
X & B
\end{array}\right]=\left[\begin{array}{ll}
A & 0 \\
X & I
\end{array}\right]\left[\begin{array}{ll}
I & 0 \\
0 & B
\end{array}\right],
$$

we see that $S=S_{1} S_{2}$. Hence $S=S^{\prime}$. Note that

$$
O_{p}(S)=\left\{s \in S \mid A_{s, 1}=A_{s, 2}=I\right\}
$$

and

$$
S / O_{p}(S) \cong \mathrm{SL}_{K}\left(V_{i-1}\right) \times \mathrm{SL}_{K}\left(V_{i-1}\right) .
$$

So $S \in \mathcal{P}$ and $n_{S}=2$.

Let $t>0$. Put $T=G_{i+t}$. We show that $V_{T}^{k}$ is block-diagonal for $S$ for $k=1,2$. For $j=1,2$, put $\gamma_{S}^{j}: S \rightarrow G_{S}^{j}, s \rightarrow A_{s, j}$. Then $G_{S}^{j}=\mathrm{SL}_{K}\left(V_{i-1}\right), \alpha_{S}^{j}=\gamma_{S}^{j} \pi_{S}^{j}$ and $\beta_{S}^{j}=\varphi_{S} \gamma_{S}^{j}$ for $j=1,2=n_{S}$. We have similar notations and results for $T$ (see the diagrams below).
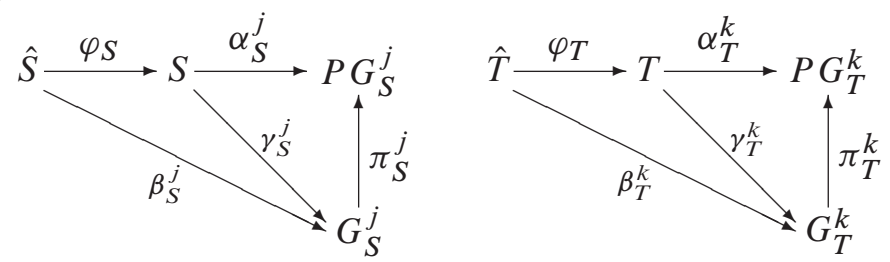

Pick $k \in\{1,2\}$. Using the notations of block-diagonality (with ' $\theta=\alpha_{T}^{k}$ '; see the diagram below), we get that

$$
\widehat{\alpha_{T}^{k}}=\varphi_{S} \gamma_{T}^{k}
$$

Note that $S$ has exactly $2^{t}$ composition factors on $V_{T}^{k}=V_{i+t-1}$ and all of them are non-trivial. The set $S_{1}$ is non-trivial and $S_{2}$ is trivial on one half of these composition factors while $S_{1}$ is trivial and $S_{2}$ is non-trivial on the other half. Let $C$ be a composition factor for $S$ on $V_{T}^{k}$. Then there exists $j \in\{1,2\}$ such that $\left[C, S_{l}\right]=0$ for $l \in\{1,2\}$ with $l \neq j$ and $C \cong V_{S}^{j}=V_{i-1}$ as $K S$-modules. Since

$$
\varphi_{S} \gamma_{S}^{j}=\beta_{S}^{j} \quad \text { and } \quad \varphi_{S} \gamma_{T}^{k}=\widehat{\alpha_{T}^{k}},
$$

$V_{T}^{k}$ is block-diagonal for $S$.

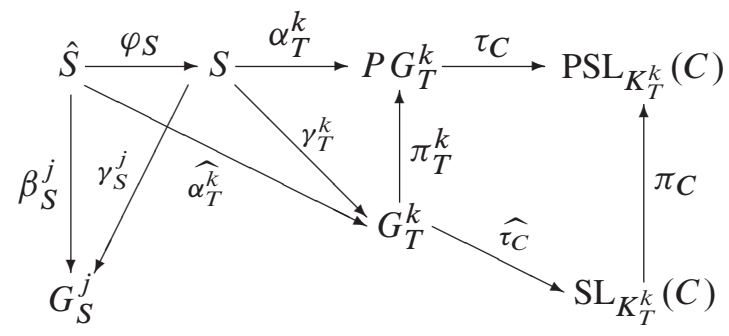


It follows from Theorem 2.1 that $G / \operatorname{LSol}(G)$ is an LFS-group of $p$-type. Note that $\operatorname{LSol}(G)=Z(G)$. Indeed, for $i \geq 1$, we have that

$$
\begin{aligned}
\operatorname{Sol}\left(G_{i}\right) & =\left\{g \in G_{i} \mid A_{g, 1}, A_{g, 2} \in Z\left(\operatorname{SL}_{K}\left(V_{i-1}\right)\right)\right\} \\
Z\left(G_{i}\right) & =\left\{g \in G_{i} \mid X_{g}=0, A_{g, 1}=A_{g, 2} \in Z\left(\operatorname{SL}_{K}\left(V_{i-1}\right)\right)\right\} \\
Z\left(G_{i}\right) & \leq Z\left(G_{i+1}\right)
\end{aligned}
$$

Hence $Z(G)=\bigcup_{i=1}^{\infty} Z\left(G_{i}\right)$. Pick $g \in \operatorname{LSol}(G)$ and $i \geq 1$ with $g \in G_{i}$. Then $g \in \operatorname{Sol}\left(G_{i}\right)$ and $g \in \operatorname{Sol}\left(G_{i+1}\right)$. Hence, as element of $G_{i}$, we get that $X_{g}=0$ and $A_{g, 1}=A_{g, 2} \in Z\left(\operatorname{SL}_{K}\left(V_{i-1}\right)\right)$. So $g \in Z\left(G_{i}\right) \leq Z(G)$. Thus $\operatorname{LSol}(G)=Z(G)$.

We now proceed with the proof of Theorem 2.1. First, we need a couple of lemmas.

Lemma 2.2. Let $K$ be a finite field with char $K=p, V$ be a finite-dimensional $K$-vector space, $G \in\left\{\operatorname{SL}_{K}(V), \operatorname{Sp}_{K}(V), \mathrm{SU}_{K}(V), \Omega_{K}(V)\right\}, P$ be a $p$-subgroup of $G, k \geq 1$ and $x \in P$ with $\operatorname{pdeg}_{V}(x)>2^{k+1}|P|^{2}+f(|P|)$ (where $f$ is the function of [5, Section 2.2]). Then there exists a p-subgroup $P^{*}$ of $G$ such that $P \leq P^{*}$ and $\operatorname{der}\left(\left\langle x^{*}\right\rangle\right) \geq k$.

Proof. The case ' $G=\mathrm{SL}_{K}(V)$ ' follows from [5, Section 5.2]. So assume that $G \neq \operatorname{SL}_{K}(V)$. By [5, Section $\left.2.3(\mathrm{c})\right]$ (with ' $G=P$ ', ' $m=|P|$ ', ' $H=\{x\}$ ' and ' $t=|P| 2^{k}$ '), there exists a $P$-invariant singular subspace $U$ of $V$ with

$$
\operatorname{pdeg}_{U}(x) \geq|P| 2^{k} .
$$

With respect to a certain basis of $V$, every $g \in N_{G}(U)$ is of the form

$$
\left[\begin{array}{ccc}
A_{g} & 0 & 0 \\
* & B_{g} & 0 \\
* & * & A_{g}^{-\sigma t}
\end{array}\right]
$$

where $\sigma \in \operatorname{Aut}(K)$ with $\sigma^{2}=1, A_{g} \in \mathrm{SL}_{K}(U)$ and $B_{g} \in \tilde{G}$ ( $\tilde{G}$ is the corresponding element in $\left.\left\{\operatorname{Sp}_{K}\left(U^{\perp} / U\right), \mathrm{SU}_{K}\left(U^{\perp} / U\right), \Omega_{K}\left(U^{\perp} / U\right)\right\}\right)$. Put

$$
\begin{aligned}
\pi_{1}: N_{G}(U) \rightarrow \mathrm{SL}_{K}(U), & & g \rightarrow A_{g}, \\
\pi_{2}: N_{G}(U) \rightarrow \tilde{G}, & & g \rightarrow B_{g}, \\
\pi: N_{G}(U) \rightarrow \mathrm{SL}_{K}(U) \times \tilde{G}, & & g \rightarrow\left(A_{g}, B_{g}\right) .
\end{aligned}
$$

Note that $\operatorname{pdeg}_{U}\left(A_{x}\right) \geq \operatorname{pdeg}_{U}(x) \geq|P| 2^{k} \geq\left|P^{\pi_{1}}\right| 2^{k}$. By [5, Section 5.2], there exists a $p$-subgroup $P_{1}$ of $\operatorname{SL}_{K}(U)$ such that $P^{\pi_{1}} \leq P_{1}$ and $\operatorname{der}\left(\left\langle\left(x^{\pi_{1}}\right)^{P_{1}}\right\rangle\right) \geq k$. 
Put $P^{*}=\left(P_{1} \times P^{\pi_{2}}\right)^{\pi^{-1}}$. Then $P^{*}$ is a $p$-group since $P^{*} / \operatorname{Ker} \pi$ and $\operatorname{Ker} \pi$ are $p$-groups and $P \leq P^{*}$ since $P^{\pi} \leq P^{\pi_{1}} \times P^{\pi_{2}} \leq P_{1} \times P^{\pi_{2}}$. Finally,

$$
\left\langle x^{P^{*}}\right\rangle^{\pi_{1}}=\left\langle\left(x^{\pi_{1}}\right)^{P_{1}}\right\rangle
$$

and so $\operatorname{der}\left(\left\langle x^{P^{*}}\right\rangle\right) \geq \operatorname{der}\left(\left\langle x^{P^{*}}\right\rangle^{\pi_{1}} \geq k\right.$.

Corollary 2.3. Lemma 2.2 remains true if

$$
G \in\left\{\mathrm{PSL}_{K}(V), \operatorname{PSp}_{K}(V), \mathrm{PSU}_{K}(V), \mathrm{P} \Omega_{K}(V)\right\} .
$$

Proof. Pick $H \in\left\{\operatorname{SL}_{K}(V), \operatorname{Sp}_{K}(V), \mathrm{SU}_{K}(V), \Omega_{K}(V)\right\}$ with $H / Z(H)=G$. Let $Q$ be a $p$-subgroup of $H$ with $Q Z(H) / Z(H)=P$ and $y \in Q$ with $y Z(H)=x$. Note that $Q \cap Z(H)=1$ and so $|Q|=|P|$. By Lemma 2.2, there exists a $p$-subgroup $Q^{*}$ of $H$ such that $Q \leq Q^{*}$ and $\operatorname{der}\left(\left\langle y Q^{*}\right\rangle\right) \geq k$. Put

$$
P^{*}=Q^{*} Z(H) / Z(H) \text {. }
$$

Then $P^{*}$ is a $p$-subgroup of $G$ containing $P$ and $\operatorname{der}\left(\left\langle x^{*}\right\rangle \geq k\right.$ since

$$
\left\langle y^{Q^{*}}\right\rangle \cong\left\langle y^{Q^{*}}\right\rangle Z(H) / Z(H)=\left\langle(y Z(H))^{Q^{*} Z(H) / Z(H)}\right\rangle=\left\langle x^{P^{*}}\right\rangle .
$$

Lemma 2.4. Let $F, K, L$ be finite fields with $F, K \subseteq L$, let $V$ be a finite-dimensional $K$-vector space, let $\alpha \in \mathrm{GL}_{K}(V)$ and $\sigma \in \operatorname{Aut}(K)$. Then

$$
\operatorname{deg}_{V^{\sigma} \otimes_{K} L}(\alpha)=\operatorname{deg}_{V^{*, \sigma} \otimes_{K} L}(\alpha)=\operatorname{deg}_{V}(\alpha)[L: F]
$$

where we view $V^{\sigma} \otimes_{K} L$ and $V^{*, \sigma} \otimes_{K} L$ as $F$-vector spaces.

Proof. Clearly, $\operatorname{deg}_{V}(\alpha)=\operatorname{deg}_{V^{\sigma}}(\alpha)$. Let $A$ be the matrix representation of $\alpha$ with respect to a certain basis of $V$. Then $A^{-t}$ is the matrix representation of $\alpha$ with respect to a certain basis of $V^{*}$. Put $n=\operatorname{dim}_{K} V$. Then for all $x \in K^{n}$, we have that $x A=x$ if and only if $x A^{-1}=x$. Hence the matrices $A-I, A^{-1}-I$ and $A^{-t}-I$ have the same rank. $\operatorname{So~}_{\operatorname{deg}_{V}}(\alpha)=\operatorname{deg}_{V^{*}}(\alpha)$. Thus we only need to prove the case ' $V \otimes_{K} L$ '. Put $W=V \otimes_{K} L$. Then

$$
\operatorname{Ker}\left(\alpha-1_{V}\right) \otimes_{K} L \subseteq \operatorname{Ker}\left(\alpha \otimes_{K} 1_{L}-1_{W}\right)
$$

and

$$
\operatorname{Im}\left(\alpha-1_{V}\right) \otimes_{K} L \subseteq \operatorname{Im}\left(\alpha \otimes_{K} 1_{L}-1_{W}\right) .
$$

Comparing the dimensions over $L$, we see that we have equality. Hence

$$
\begin{aligned}
\operatorname{deg}_{W}(\alpha) & =\operatorname{dim}_{F}\left(\operatorname{Im}\left(\alpha \otimes_{K} 1_{L}-1_{W}\right)\right) \\
& =\operatorname{dim}_{F}\left(\operatorname{Im}\left(\alpha-1_{V}\right) \otimes_{K} L\right) \\
& =\operatorname{dim}_{K}\left(\operatorname{Im}\left(\alpha-1_{V}\right)\right)[L: F] \\
& =\operatorname{deg}_{V}(\alpha)[L: F]
\end{aligned}
$$

which proves the lemma. 
Now we can prove Theorem 2.1.

Proof of Theorem 2.1. First, we show that $G / \operatorname{LSol}(G)$ is simple and that

$$
[G, \operatorname{LSol}(G)] \leq O_{p}(G) .
$$

Pick $x \in G \backslash \operatorname{LSol}(G)$. Suppose that $x \in \operatorname{Sol}(R)$ for all $R \in \delta(x)$. Let $F$ be a finite subgroup of $\left\langle x^{G}\right\rangle$. Pick $R \in \delta(x)$ such that $F \leq\left\langle x^{R}\right\rangle$. Then $F \leq \operatorname{Sol}(R)$. So $\left\langle x^{G}\right\rangle$ is locally solvable and $\left\langle x^{G}\right\rangle \leq \operatorname{LSol}(G)$, a contradiction. Hence there exists $R \in \delta(x)$ with $x \notin \operatorname{Sol}(R)$. Pick $S \in \delta(R)$. Then $x \notin \operatorname{Sol}(S)$. Put $T=F_{S}$. Put $x=s_{1} \ldots s_{n_{S}}=t_{1} \ldots t_{n_{T}}$ where $s_{i} \in S_{i}$ for $i=1, \ldots, n_{S}$ and $t_{j} \in T_{j}$ for $j=1, \ldots, n_{T}$. Since $x \notin \operatorname{Sol}(S)$, there exists $1 \leq i \leq n_{S}$ with $s_{i} \notin \operatorname{Sol}\left(S_{i}\right)$. Pick $1 \leq j \leq n_{T}$. Since $\left[V_{T}^{j}, S_{i}^{*}\right] \neq 0$ and $S_{i}^{*}$ is perfect, there exists a composition factor $C$ for $\hat{S}$ on $V_{T}^{j}$ with

$$
\left[C, S_{i}^{*}\right] \neq 0 .
$$

By block-diagonality, there exists $\sigma \in \operatorname{Aut}\left(K_{S}^{i}\right)$ such that

$$
C \cong\left(V_{S}^{i}\right)^{\sigma} \otimes_{K_{S}^{i}}\left\langle K_{S}^{i}, K_{T}^{j}\right\rangle
$$

or

$$
C \cong\left(V_{S}^{i}\right)^{*, \sigma} \otimes_{K_{S}^{i}}\left\langle K_{S}^{i}, K_{T}^{j}\right\rangle
$$

as $K_{T}^{j} \hat{S}$-modules. Pick $y \in \hat{S}$ with $y^{\varphi_{S}}=x$. We use the notations of the definition of block-diagonality with ' $\theta=\alpha_{T}^{j}$, (see the diagram below). Since $s_{i} \notin \operatorname{Sol}\left(S_{i}\right)$, $1 \neq x^{\alpha_{S}^{i}}=y^{\varphi_{S} \alpha_{S}^{i}}=y^{\beta_{S}^{i} \pi_{S}^{i}}$ and so $y$ does not act like a scalar on $V_{S}^{i}$. Hence $y$ does not act like a scalar on $C$, nor on $V_{T}^{j}$. So

$$
1 \neq y^{\alpha_{T}^{j} \pi_{T}^{j}}=y^{\varphi_{S} \alpha_{T}^{j}}=x^{\alpha_{T}^{j}} .
$$

Hence $t_{j} \notin \operatorname{Sol}\left(T_{j}\right)$.
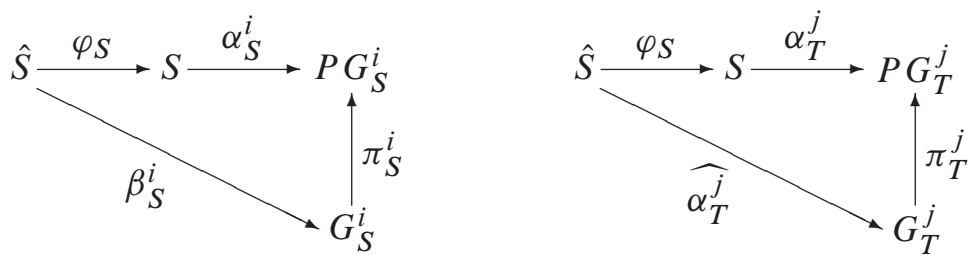

By [1, Section 1.6 and $1.7(b)]$,

$$
\left\langle x^{T}\right\rangle \operatorname{Sol}(T) / \operatorname{Sol}(T)=P G_{T}^{1} \times \cdots \times P G_{T}^{n_{T}}
$$

and so $\left\langle x^{T}\right\rangle \operatorname{Sol}(T)=T$. Taking perfect parts, we get that

$$
T=T^{\infty}=\left\langle x^{T}\right\rangle^{\infty} \leq\left\langle x^{T}\right\rangle
$$


and so $\left\langle x^{T}\right\rangle=T$. Hence

$$
S \leq\left\langle x^{G}\right\rangle \quad \text { for all } S \in \delta(R) .
$$

Thus $\left\langle x^{G}\right\rangle=G$ and $G / \operatorname{LSol}(G)$ is simple.

Let $F$ be a finite subgroup of $G$ and $H$ be a finite subgroup of $\operatorname{LSol}(G)$. Pick $S \in \delta(\langle F, H\rangle)$. Then $H \leq S \cap \operatorname{LSol}(G) \leq \operatorname{Sol}(S)$ and so

$$
[F, H] \leq[S, \operatorname{Sol}(S)] \leq O_{p}(S) .
$$

Hence $[G, \operatorname{LSol}(G)]$ is a $p$-group and so $[G, \operatorname{LSol}(G)] \leq O_{p}(G)$.

Next, we show that $G / \operatorname{LSol}(G)$ is infinite and not finitary by constructing a non-solvable $p$-subgroup. Condition (ii) (c) is crucial here.

Pick $x \in G$ such that $|x|$ is a power of $p$ and $x \notin \operatorname{LSol}(G)$. We prove two claims needed to construct this non-solvable $p$-subgroup.

Claim 1. For all $S \in \delta(x)$ and $k \geq 1$, there exists $T_{k} \in \delta(S)$ with

$$
\operatorname{pdeg}_{V_{T_{k}}^{j}}(x) \geq 2^{k-1} \text { for } j=1, \ldots, n_{T_{k}} \text {. }
$$

Pick $S \in \delta(x)$. We induct on $k$. Pick $T_{0} \in \delta(S)$ with $x \notin \operatorname{Sol}\left(T_{0}\right)$. Suppose now that $k \geq 1$. Put $T_{k}=F_{T_{k-1}}$. Pick $y \in \widehat{T_{k-1}}$ such that $|y|$ is a power of $p$ and $y^{\varphi_{T_{k-1}}}=x$. For $i=1, \ldots, n_{T_{k-1}}$,

$$
y^{\beta_{T_{k-1}}^{i} \pi_{T_{k-1}}^{i}}=y^{\varphi_{T_{k-1}} \alpha_{T_{k-1}}^{i}}=x^{\alpha_{T_{k-1}}^{i}}
$$

and so

Similarly,

$$
\operatorname{pdeg}_{V_{T_{k-1}}^{i}}(x)=\operatorname{pdeg}_{V_{T_{k-1}}^{i}}(y) .
$$

$$
\operatorname{pdeg}_{V_{T_{k}}^{j}}(x)=\operatorname{pdeg}_{V_{T_{k}}^{j}}(y) \text { for } j=1, \ldots, n_{T_{k}} .
$$

Let $1 \leq j \leq n_{T_{k}}$. Using the definition of block-diagonality and Lemma 2.4, we get that

$$
\operatorname{pdeg}_{V_{T_{k}}^{j}}(y) \geq \sum_{i=1}^{n_{T_{k-1}}} n_{T_{k-1} T_{k}}^{i j} \operatorname{pdeg}_{V_{T_{k-1}}^{i}}(y) \operatorname{dim}_{K_{T_{k}}^{j}}\left\langle K_{T_{k-1}}^{i}, K_{T_{k}}^{j}\right\rangle .
$$

If $k=1$, then there exists $1 \leq i \leq n_{T_{0}}$ with $x \notin \operatorname{Ker} \alpha_{T_{0}}^{i}$ since $x \notin \operatorname{Sol}\left(T_{0}\right)$ and so

$$
\operatorname{pdeg}_{V_{T_{0}}^{i}}(x) \geq 1 \text {. }
$$

Hence

$$
\operatorname{pdeg}_{V_{T_{1}}^{j}}(y) \geq \sum_{i=1}^{n_{T_{0}}} n_{T_{0} T_{1}}^{i j} \operatorname{pdeg}_{V_{T_{0}}^{i}}(y) \operatorname{dim}_{K_{T_{1}}^{j}}\left\langle K_{T_{0}}^{i}, K_{T_{1}}^{j}\right\rangle \geq 1 .
$$


If $k \geq 2$, we can use induction to get that

$$
\begin{aligned}
\operatorname{pdeg}_{V_{T_{k}}^{j}}(y) & \geq \sum_{i=1}^{n_{T_{k-1}}} n_{T_{k-1} T_{k}}^{i j} \operatorname{pdeg}_{V_{T_{k-1}}^{i}}(y) \operatorname{dim}_{K_{T_{k}}^{j}}\left\langle K_{T_{k-1}}^{i}, K_{T_{k}}^{j}\right\rangle \\
& \geq 2^{k-2} \sum_{i=1}^{n_{T_{k-1}}} n_{T_{k-1} T_{k}}^{i j} \operatorname{dim}_{K_{T_{k}}^{j}}\left\langle K_{T_{k-1}}^{i}, K_{T_{k}}^{j}\right\rangle \\
& \geq 2^{k-2} \cdot 2=2^{k-1} .
\end{aligned}
$$

This proves Claim 1 .

Claim 2. There exist finite $p$-subgroups $P_{1}, P_{2}, \ldots$ of $G$ such that

$$
x \in P_{1} \leq P_{2} \leq \cdots
$$

and $\operatorname{der}\left(\left\langle x^{P_{k}}\right\rangle \operatorname{LSol}(G) / \operatorname{LSol}(G)\right) \geq k$ for $k=1,2, \ldots$

We induct on $k$. For $k=1$, we put $P_{1}=\langle x\rangle$ since $x \notin \operatorname{LSol}(G)$. So suppose $k \geq 2$. Pick $S \in \delta\left(P_{k-1}\right)$. By Claim 1, there exists $T \in \delta(S)$ such that

$$
\operatorname{pdeg}_{V_{T}^{1}}(x)>2^{k+1}\left|P_{k-1}\right|^{2}+f\left(\left|P_{k-1}\right|\right)
$$

( $f$ is the function of Lemma 2.2). Note that $\left|P_{k-1}^{\alpha_{T}^{1}}\right| \leq\left|P_{k-1}\right|$. By Corollary 2.3, there exists a $p$-subgroup $P^{*}$ of $P G_{T}^{1}$ such that

$$
P_{k-1}^{\alpha_{T}^{1}} \leq P^{*} \quad \text { and } \quad \operatorname{der}\left(\left\langle\left(x^{\alpha_{T}^{1}}\right)^{P^{*}}\right\rangle\right) \geq k .
$$

Note that $O_{p}(T) \leq \operatorname{Ker} \alpha_{T}^{1}$. Hence there exists a $p$-subgroup $P_{k}$ of $T$ such that

$$
P_{k-1}, O_{p}(T) \leq P_{k} \quad \text { and } \quad P_{k}^{\alpha_{T}^{1}}=P^{*} .
$$

Then

$$
\left\langle x^{P_{k}}\right\rangle^{\alpha_{T}^{1}}=\left\langle\left(x^{\alpha_{T}^{1}}\right)^{P_{k}^{\alpha}}\right\rangle=\left\langle\left(x^{\alpha_{T}^{1}}\right)^{P^{*}}\right\rangle
$$

and so $\operatorname{der}\left(\left\langle x^{P_{k}}\right\rangle^{\alpha_{T}^{1}}\right) \geq k$. Note that

$$
\begin{aligned}
M:=\left\langle x^{P_{k}}\right\rangle \cap \operatorname{LSol}(G) & =\left\langle x^{P_{k}}\right\rangle \cap T \cap \operatorname{LSol}(G) \\
& \leq\left\langle x^{P_{k}}\right\rangle \cap \operatorname{Sol}(T) \leq\left\langle x^{P_{k}}\right\rangle \cap \operatorname{Ker} \alpha_{T}^{1}:=N
\end{aligned}
$$

and so

$$
\left\langle x^{P_{k}}\right\rangle^{\alpha_{T}^{1}} \cong\left\langle x^{P_{k}}\right\rangle /\left\langle x^{P_{k}}\right\rangle \cap \operatorname{Ker} \alpha_{T}^{1}=\left\langle x^{P_{k}}\right\rangle / N \cong\left(\left\langle x^{P_{k}}\right\rangle / M\right) /(N / M) .
$$


Hence

$$
\begin{aligned}
\operatorname{der}\left(\left\langle x^{P_{k}}\right\rangle \operatorname{LSol}(G) / \operatorname{LSol}(G)\right) & =\operatorname{der}\left(\left\langle x^{P_{k}}\right\rangle /\left\langle x^{P_{k}}\right\rangle \cap \operatorname{LSol}(G)\right) \\
& \geq \operatorname{der}\left(\left\langle x^{P_{k}}\right\rangle^{\alpha_{T}^{1}}\right) \geq k
\end{aligned}
$$

This proves Claim 2.

Let $P_{1}, P_{2}, \ldots$ be as in Claim 2. Put $P=\bigcup_{k=1}^{\infty} P_{k}$. Then $P$ is a $p$-subgroup of $G, x \in P$ but $\left\langle x^{P}\right\rangle \operatorname{LSol}(G) / \operatorname{LSol}(G)$ is not solvable. $\operatorname{So} G / \operatorname{LSol}(G)$ is infinite. By [6, Proposition 1], $G / \operatorname{LSol}(G)$ is not finitary.

Finally, we show that $G / \operatorname{LSol}(G)$ is of $p$-type. By [2, Section 2.3], there exists an odd prime $q \neq p$ with $2 \alpha(q)+1<q$ where $\alpha(q)$ is the order of $p$ modulo $q$. By the first claim, there exists $S \in \mathcal{S}$ with

$$
\operatorname{dim}_{K_{S}^{1}} V_{S}^{1}>2 \alpha(q)(p-1)+1 .
$$

Pick $g \in G_{S}^{1}$ as in [2, Section 2.4], so

$$
|g|=q, \quad g \notin Z\left(\mathrm{GL}_{K_{S}^{1}}\left(V_{S}^{1}\right)\right),
$$

the matrix representation $B$ of $g$ with respect to a certain $K_{S}^{1}$-basis for $V_{S}^{1}$ has all its entries in $\operatorname{GF}(p), 1$ is a root of the characteristic polynomial $f_{K_{S}^{1}}(g)$ of $g$, $f_{K_{S}^{1}}(g)$ has at most $2 \alpha(q)+1$ different roots over $\overline{\mathrm{GF}(p)}$ and

$$
f_{K_{S}^{1}}(B)=f_{K_{S}^{1}}\left(B^{-1}\right) .
$$

Pick $y \in S_{1}^{*}$ such that $|y|$ is a power of $q$ and $y^{\beta_{S}^{1}}=g$. Put $x=y^{\varphi_{S}}$. By [2, Section 2.4(b)],

$$
1 \neq g^{\pi_{S}^{1}}=y^{\beta_{S}^{1} \pi_{S}^{1}}=y^{\varphi_{S} \alpha_{S}^{1}}=x^{\alpha_{S}^{1}} .
$$

So we have $x \notin \operatorname{Sol}(S) \leq \operatorname{Ker} \alpha_{S}^{1}$ and $x \notin \operatorname{LSol}(G)$. Let $\tilde{Q}$ be a finite $q$-subgroup of $G / \operatorname{LSol}(G)$ with $x \operatorname{LSol}(G) \in \tilde{Q}$. Then $\tilde{Q}=Q \operatorname{LSol}(G) / \operatorname{LSol}(G)$ for some finite $q$-subgroup $Q$ of $G$ with $x \in Q$. Pick $T \in \mathcal{S}(S, Q)$ such that $V_{T}^{j}$ is blockdiagonal for $S$ for $j=1, \ldots, n_{T}$. Let $\varphi: \hat{S} \rightarrow \hat{T}$ be the unique homomorphism with $\varphi \varphi_{T}=\varphi_{S}$. Pick $1 \leq j \leq n_{T}$. We use the notations of the definition of blockdiagonality with ' $\theta=\alpha_{T}^{\bar{j}}$ ' (see the diagram below). Note that

$$
\varphi \beta_{T}^{j} \pi_{T}^{j}=\varphi \varphi_{T} \alpha_{T}^{j}=\varphi_{S} \alpha_{T}^{j}=\widehat{\alpha_{T}^{j}} \pi_{T}^{j} .
$$

So $\widehat{\alpha_{T}^{j}}=\varphi \beta_{T}^{j}$ since $\widehat{\alpha_{T}^{j}}$ is unique with this property. Put $\tilde{y}=y^{\varphi \beta_{T}^{j}}$. Let $f_{K_{T}^{j}}(\tilde{y})$ be the characteristic polynomial of $\tilde{y}$ and $f_{K_{S}^{1}}(g)$ the characteristic polynomial of $g$. Using block-diagonality, [2, Section 2.5] and [2, Section 2.4], we see that

$$
f_{K_{T}^{j}}(\tilde{y})=(X-1)^{m} f_{K_{S}^{1}}(g)^{n}
$$


for some $m, n \geq 0$ and $\tilde{y}^{q}$ is trivial on every composition factor for $\hat{S}$ on $V_{T}^{j}$. So $\tilde{y}^{q}=1$ since $|\tilde{y}|$ is a power of $q$ and $q \neq p$. Note that

$$
\tilde{y}^{\pi_{T}^{j}}=y^{\varphi \beta_{T}^{j} \pi_{T}^{j}}=y^{\varphi \varphi_{T} \alpha_{T}^{j}}=y^{\varphi_{S} \alpha_{T}^{j}}=x^{\alpha_{T}^{j}} .
$$

Hence there exists a $q$-subgroup $Q_{*}$ of $G_{T}^{j}$ such that $\tilde{y} \in Q_{*}$ and $\ln Q_{*}^{\pi_{T}^{j}}=Q^{\alpha_{T}^{j}}$.
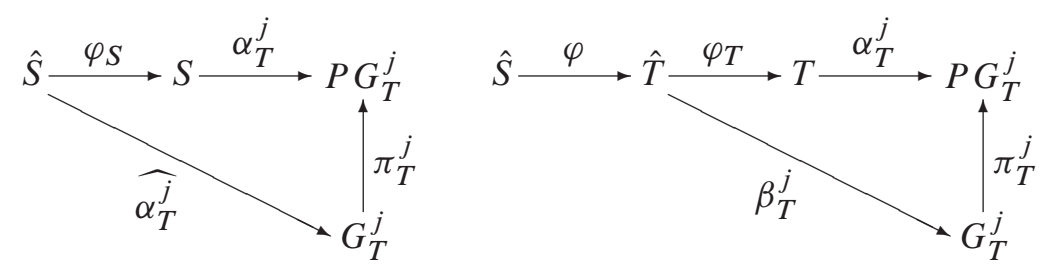

By [2, Section $2.4(\mathrm{~d})(\mathrm{e})]$, the polynomial $f_{K_{T}^{j}}(\tilde{y})$ has at most $2 \alpha(q)+1$ different roots over $\overline{\mathrm{GF}(p)}$. Hence $X^{q}-1$ does not divide $f_{K_{T}^{j}}(\tilde{y})$ since $X^{q}-1$ has $q$ different roots over $\overline{\mathrm{GF}(p)}$ and $2 \alpha(q)+1<q$. If $\tilde{y}=1$, then $\left\langle\tilde{y} Q_{*}\right\rangle=1$ and so abelian. If $|\tilde{y}|=q$, then $\left\langle\tilde{y}_{*}\right\rangle$ is abelian by [2, Section 2.6]. Hence

$$
\left\langle\tilde{y}_{*}\right\rangle^{\pi_{T}^{j}}=\left\langle\left(\tilde{y}^{\pi_{T}^{j}}\right)^{Q_{*}^{\pi_{T}^{j}}}\right\rangle=\left\langle\left(x^{\alpha_{T}^{j}}\right)^{Q^{\alpha_{T}^{j}}}\right\rangle=\left\langle x{ }^{Q}\right\rangle^{\alpha_{T}^{j}}
$$

is abelian. So $\left\langle x^{Q}\right\rangle^{\alpha_{T}^{j}}$ is abelian for $j=1, \ldots, n_{T}$. Since

$$
\bigcap_{j=1}^{n_{T}} \operatorname{Ker} \alpha_{T}^{j}=\operatorname{Sol}(T),
$$

we get that $\left\langle x^{Q}\right\rangle \operatorname{Sol}(T) / \operatorname{Sol}(T)$ is abelian. If $X \subseteq T$, we put

$$
\bar{X}=X O_{p}(T) / O_{p}(T) \text {. }
$$

Then

$$
\left\langle x^{Q}\right\rangle \operatorname{Sol}(T) / \operatorname{Sol}(T) \cong\left\langle\bar{x}^{\bar{Q}}\right\rangle \overline{\operatorname{Sol}(T)} / \overline{\operatorname{Sol}(T)} \cong\left\langle\bar{x}^{\bar{Q}}\right\rangle /\left\langle\bar{x}^{\bar{Q}}\right\rangle \cap \overline{\operatorname{Sol}(T)} .
$$

Note that $\overline{\operatorname{Sol}(T)}=\operatorname{Sol}(\bar{T})=Z(\bar{T})$ and so $\langle\bar{x} \bar{Q}\rangle$ is solvable of length at most two. But

$$
\left\langle\bar{x}^{Q}\right\rangle=\overline{\left\langle x^{Q}\right\rangle}=\left\langle x^{Q}\right\rangle O_{p}(T) / O_{p}(T) \cong\left\langle x^{Q}\right\rangle /\left\langle x^{Q}\right\rangle \cap O_{p}(T)=\left\langle x^{Q}\right\rangle
$$

since $\left\langle x^{Q}\right\rangle \cap O_{p}(T)=1$. Hence $\left\langle x^{Q}\right\rangle$ is solvable of length at most two and so

$$
\left\langle x^{Q}\right\rangle \operatorname{LSol}(G) / \operatorname{LSol}(G)=\left\langle(x \operatorname{LSol}(G))^{\tilde{Q}}\right\rangle
$$

is solvable of length at most two. Since $G / \operatorname{LSol}(G)$ is locally finite, it follows that the group $\left\langle(x \operatorname{LSol}(G))^{\tilde{Q}}\right\rangle$ is solvable for all $q$-subgroups $\tilde{Q}$ of $G / \operatorname{LSol}(G)$ with $x \operatorname{LSol}(G) \in \tilde{Q}$. By [2, Section 3.1 (b)], G/LSol(G) is of $p^{\prime}$-type for some 
prime $p^{\prime}$. Note that $\{S \operatorname{LSol}(G) / \operatorname{LSol}(G) \mid S \in \delta\}$ is a local system for the quotient $G / \operatorname{LSol}(G)$. Moreover, if $S \in \mathcal{8}$, then $S / \operatorname{Sol}(S) \cong P G_{S}^{1} \times \cdots \times P G_{S}^{n}$ and so all non-abelian composition factors of $S$ are classical groups in characteristic $p$. The same is true for $S \operatorname{LSol}(G) / \operatorname{LSol}(G)$ since

$$
S \operatorname{LSol}(G) / \operatorname{LSol}(G) \cong S / S \cap \operatorname{LSol}(G)
$$

and $S \cap \operatorname{LSol}(G) \leq \operatorname{Sol}(S)$. By [5, Section $2.15(\mathrm{~d})], G / \operatorname{LSol}(G)$ has a Kegel cover all of whose factors are classical groups in characteristic $p$. It follows now from the definition of $p^{\prime}$-type that $p^{\prime}=p$.

\section{General construction of LFS-groups of $p$-type}

In this section, we give a general construction of LFS-groups of $p$-type, which will be a special case of Theorem 2.1: $S=G_{S}^{1} \times \cdots \times G_{S}^{n_{S}}$ for all $S \in 8$. Note that $\operatorname{LSol}(G)=Z(G)$ in this special case. Indeed, pick $x \in \operatorname{LSol}(G)$ and $g \in G$. Then there exists $S \in \mathcal{S}$ with $x, g \in S$. Hence $x \in S \cap \operatorname{LSol}(G) \leq \operatorname{Sol}(S)=Z(S)$ and so $x g=g x$. Thus $x \in Z(G)$ and $\operatorname{LSol}(G) \leq Z(G)$. Hence $\operatorname{LSol}(G)=Z(G)$.

We start with an example that illustrates the general method. Let $K$ be a finite field with char $K=p$ and $V_{n}$ be a $K$-vector space with $\operatorname{dim}_{K} V_{n} \geq 2$ for all $n \geq 1$. Put $V=V_{1} \otimes_{K} V_{2} \otimes_{K} V_{3} \otimes_{K} \cdots$. For $n \geq 1$, put $G_{n}=\mathrm{SL}_{K}\left(V_{1} \otimes_{K} \cdots \otimes_{K} V_{n}\right)$. We can view $G_{n}$ as a subgroup of $\mathrm{GL}_{K}(V)$ via the embedding $G_{n} \rightarrow \mathrm{GL}_{K}(V)$, $g \rightarrow g \otimes_{K} 1_{W_{n}}$ where $W_{n}=V_{n+1} \otimes_{K} V_{n+2} \otimes_{K} \cdots$. Note that the composition factors of $G_{n}$ on $V$ are isomorphic to $V_{1} \otimes_{K} \cdots \otimes_{K} V_{n}$. So we have block-diagonal embeddings. Put $G=\bigcup_{n \geq 1} G_{n}$. Then $G / Z(G)$ is an LFS-group of $p$-type.

We can generalize this as follows.

Let $I$ be an infinite set, $K_{i}$ be a finite subfield of $\overline{\operatorname{GF}(p)}$ and $V_{i}$ be a finitedimensional $K_{i}$-vector space of dimension at least two for all $i \in I$.

For $\emptyset \neq J \subseteq I$, put $K_{J}=\left\langle K_{j} \mid j \in J\right\rangle$ (the field generated by the $K_{j}$ with $j \in J)$ and $V_{J}=\otimes_{j \in J}\left(V_{j} \otimes_{K_{j}} K_{J}\right)$ where the tensor product is taken over $K_{J}$. We put $K=K_{I}$ and $V=V_{I}$.

Let $\emptyset \neq J \subseteq L \subseteq I$. We view $V_{L}$ as $\left(V_{J} \otimes_{K_{J}} K_{L}\right) \otimes_{K_{L}}\left(V_{L \backslash J} \otimes_{K_{L \backslash J}} K_{L}\right)$. Put $\theta_{J L}: \mathrm{GL}_{K_{J}}\left(V_{J}\right) \rightarrow \mathrm{GL}_{K_{L}}\left(V_{L}\right)$ by

$$
\left(\left(v \otimes_{K_{J}} 1_{K_{L}}\right) \otimes_{K_{L}}\left(w \otimes_{K_{L \backslash J}} 1_{K_{L}}\right)\right)^{g^{\theta} J L}=\left(v^{g} \otimes_{K_{J}} 1_{K_{L}}\right) \otimes_{K_{L}}\left(w \otimes_{K_{L \backslash J}} 1_{K_{L}}\right)
$$

for all $v \in V_{J}, w \in V_{L \backslash J}$ and $g \in \mathrm{GL}_{K_{J}}\left(V_{J}\right)$. Then $\theta_{J L}$ is a monomorphism. Note that

$$
\mathrm{SL}_{K_{J}}\left(V_{J}\right)^{\theta_{J L}} \leq \mathrm{SL}_{K_{L}}\left(V_{L}\right)
$$

if $L$ is finite. One easily checks that $\theta_{J L} \theta_{L N}=\theta_{J N}$ if $\emptyset \neq J \subseteq L \subseteq N \subseteq I$. 
For every non-empty, finite subset $J$ of $I$, we put

$$
G_{J}=\operatorname{SL}_{K_{J}}\left(V_{J}\right)^{\theta_{J I}} \leq \mathrm{GL}_{K}(V) .
$$

If $J, L$ are non-empty, finite subsets of $I$ with $J \subseteq L$, then

$$
G_{J}=\mathrm{SL}_{K_{J}}\left(V_{J}\right)^{\theta_{J I}}=\mathrm{SL}_{K_{J}}\left(V_{J}\right)^{\theta_{J L} \theta_{L I}} \leq \mathrm{SL}_{K_{L}}\left(V_{L}\right)^{\theta_{L I}}=G_{L}
$$

and $Z\left(G_{J}\right) \leq Z\left(G_{L}\right)$.

Put $G=\bigcup\left\{G_{J} \mid \emptyset \neq J \subseteq I, J\right.$ finite $\}$. Then $G$ is an infinite, locally finite group and $\left\{G_{J} \mid \varnothing \neq J \subseteq I, J\right.$ finite, $\left.\operatorname{dim}_{K_{J}} V_{J} \geq 3\right\}$ is a local system for $G$, contained in $\mathcal{P}$.

Let $J$ be a non-empty, finite subset of $I$ such that $\operatorname{dim}_{K_{J}} V_{J} \geq 3$. Pick a finite subset $L$ of $I$ containing $J$. Note that we view $V_{L}$ as

$$
\left(V_{J} \otimes_{K_{J}} K_{L}\right) \otimes_{K_{L}}\left(V_{L \backslash J} \otimes_{K_{L \backslash J}} K_{L}\right) .
$$

Hence $\left[V_{L}, G_{J}\right] \neq 0$. Let $\left\{f_{1}, \ldots, f_{n}\right\}$ be a $K_{L \backslash J}$-basis for $V_{L \backslash J}$. Then

$$
V_{L}=\bigoplus_{i=1}^{n}\left(V_{J} \otimes_{K_{J}} K_{L}\right) \otimes_{K_{L}}\left(f_{i} \otimes_{K_{L \backslash J}} 1_{K_{L}}\right) .
$$

Note that $V_{J} \otimes_{K_{J}} K_{L}$ is an irreducible $K_{L} G_{J}$-module. Hence every composition factor for $G_{J}$ on $V_{L}$ is isomorphic to $V_{J} \otimes_{K_{J}} K_{L}$ (and so non-trivial) and $V_{L}$ is block-diagonal for $G_{J}$. Also $G_{J}$ has $\operatorname{dim}_{K_{L \backslash J}} V_{L \backslash J}$ composition factors on $V_{L}$. So $G_{J}$ has at least two non-trivial composition factors on $V_{L}$ if $|L \backslash J| \geq 1$. By Theorem 2.1, $G / Z(G)$ is an LFS-group of $p$-type.

\section{Bibliography}

[1] S. Delcroix, Block-diagonality of LFS-groups of p-type, J. Algebra 315 (2007), no. 1, 419-453.

[2] S. Delcroix, Local characterization of non-finitary LFS-groups, J. Group Theory 11 (2008), no. 4, 537-544.

[3] S. Delcroix and U. Meierfrankenfeld, Locally finite simple groups of 1-type, J. Algebra 247 (2002), no. 2, 728-746.

[4] O. Kegel and B. A. F. Wehrfritz, Locally Finite Groups, North-Holland, Amsterdam, 1973.

[5] U. Meierfrankenfeld, Non-finitary locally finite simple groups, in: Finite and Locally Finite Groups (Istanbul 1994), Kluwer Academic Publishers, Dordrecht (1995), 189212.

[6] U. Meierfrankenfeld, R.E. Phillips and O. Puglisi, Locally solvable finitary linear groups, J. Lond. Math. Soc. (2) 47 (1991), 31-40. 
Received February 13, 2012; revised January 2, 2013.

\section{Author information}

Stefaan Delcroix, Department of Mathematics, California State University Fresno, 5245 N Backer Avenue M/S PB108, Fresno, Ca 93740, USA.

E-mail: sdelcroix@csufresno.edu 